Acta Crystallographica Section E

Structure Reports

Online

ISSN 1600-5368

\section{3,3-Bis(methylsulfanyl)-1-(4-nitrophenyl)- prop-2-en-1-one}

\section{Guan-Neng Yu, ${ }^{\mathrm{a}}$ Jian-Hui Xia, ${ }^{\mathrm{a}}$ Zhao-Hui Xu, ${ }^{\mathrm{a}}$ Li-Ben Wang $^{\mathrm{b}}$ and Chu-Yi Yu${ }^{\mathrm{b} *}$}

${ }^{a}$ College of Chemistry and Chemical Engineering, Jiang Xi Normal University, Nanchang, Jiang Xi 330022, People's Republic of China, and Beijing National Laboratory for Molecular Science (BNLMS), CAS Key Laboratory of Molecular Recognition and Function, Institute of Chemistry, Chinese Academy of Sciences, Beijing 100190, People's Republic of China

Correspondence e-mail: yucy@iccas.ac.cn

Received 16 March 2013; accepted 27 May 2013

Key indicators: single-crystal X-ray study; $T=173 \mathrm{~K}$; mean $\sigma(\mathrm{C}-\mathrm{C})=0.004 \AA$; $R$ factor $=0.055 ; w R$ factor $=0.115 ;$ data-to-parameter ratio $=17.4$.

In the title compound, $\mathrm{C}_{11} \mathrm{H}_{11} \mathrm{NO}_{3} \mathrm{~S}_{2}$, the $\mathrm{S}-\mathrm{Csp}{ }^{2}$ bonds are shorter [1.746 (3) and $1.750(2) \AA]$ than the $\mathrm{S}-\mathrm{CH}_{3}$ bonds [1.794 (3) and $1.806(3) \AA]$, which we attribute to $d-\pi$ interactions between the $\mathrm{S}$ atoms and the $\mathrm{C}=\mathrm{C}$ bond. The 1,1-bis(methylsulfanyl)-3-oxopropylene fragment and the 4-nitrophenyl group are both almost planar, with the largest deviations from their mean planes being 0.053 (1) and 0.017 (2) $\AA$, respectively. The dihedral angle between the two planes is $35.07(7)^{\circ}$. Molecules in the crystal are linked into a three-dimensional network by $\mathrm{C}-\mathrm{H} \cdots \mathrm{S}$ and $\mathrm{C}-\mathrm{H} \cdots \mathrm{O}$ hydrogen bonds.

\section{Related literature}

For the synthesis of the title compound, see: Huang \& Liu (1989). For applications, see: Barun et al. (2000); Kuettel et al. (2007). For general background on ketene aminals, see: Huang \& Wang (1994).

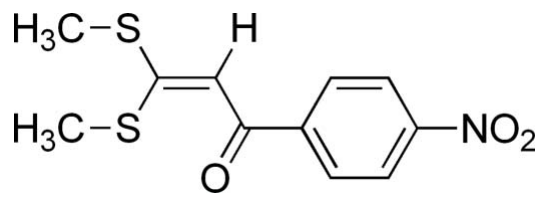

\section{Experimental}

Crystal data

$\mathrm{C}_{11} \mathrm{H}_{11} \mathrm{NO}_{3} \mathrm{~S}_{2} \quad M_{r}=269.33$
Triclinic, $P \overline{1}$

$a=7.917$ (2) $\AA$

$b=8.739$ (2) $\AA$

$c=9.574(3) \AA$

$\alpha=70.415(13)^{\circ}$

$\beta=81.985(14)^{\circ}$

$\gamma=73.283(13)^{\circ}$

\section{Data collection \\ Rigaku Saturn724+ CCD diffractometer \\ $T_{\min }=0.581, T_{\max }=1.000$ \\ Refinement \\ $R\left[F^{2}>2 \sigma\left(F^{2}\right)\right]=0.055$ \\ $w R\left(F^{2}\right)=0.115$ \\ $S=1.15$ \\ 2719 reflections}

Absorption correction: multi-scan (CrystalClear; Rigaku, 2007)

$V=597.0(3) \AA^{3}$

$Z=2$

Mo $K \alpha$ radiation

$\mu=0.44 \mathrm{~mm}^{-1}$

$T=173 \mathrm{~K}$

$0.27 \times 0.24 \times 0.05 \mathrm{~mm}$

7843 measured reflections 2719 independent reflections 2394 reflections with $I>2 \sigma(I)$ $R_{\text {int }}=0.053$

Table 1

Hydrogen-bond geometry $\left(\AA{ }^{\circ}\right)$.

\begin{tabular}{lllll}
\hline$D-\mathrm{H} \cdots A$ & $D-\mathrm{H}$ & $\mathrm{H} \cdots A$ & $D \cdots A$ & $D-\mathrm{H} \cdots A$ \\
\hline $\mathrm{C} 11-\mathrm{H} 11 \cdots \mathrm{S}^{\text {i }}$ & 0.95 & 2.93 & $3.614(3)$ & 130 \\
$\mathrm{C} 8-\mathrm{H} 8 \cdots$ O3 $^{\text {ii }}$ & 0.95 & 2.63 & $3.204(3)$ & 119 \\
$\mathrm{C} 2-\mathrm{H} 2 B \cdots \mathrm{O} 3^{\text {iii }}$ & 0.98 & 2.68 & $3.297(4)$ & 122 \\
$\mathrm{C} 10-\mathrm{H} 10 \cdots \mathrm{O} 1^{\text {iv }}$ & 0.95 & 2.66 & $3.602(3)$ & 171 \\
$\mathrm{C} 1-\mathrm{H} 1 C \cdots \mathrm{O} 1^{\text {v }}$ & 0.98 & 2.59 & $3.551(3)$ & 167 \\
$\mathrm{C} 7-\mathrm{H} 7 \cdots \mathrm{O} 2^{\text {vi }}$ & 0.95 & 2.55 & $3.499(3)$ & 179 \\
\hline
\end{tabular}

Symmetry codes: (i) $-x+2,-y+1,-z$; $\quad$ (ii) $-x+1,-y+2,-z+1$; $\quad$ (iii) $-x+2,-y+1,-z+1$; (iv) $x-1, y, z$; (v) $x, y-1, z$; (vi) $x+1, y, z$.

Data collection: CrystalClear (Rigaku, 2007); cell refinement: CrystalClear; data reduction: CrystalClear; $\operatorname{program}(\mathrm{s})$ used to solve structure: SHELXS97 (Sheldrick, 2008); program(s) used to refine structure: SHELXL97 (Sheldrick, 2008); molecular graphics: OLEX2 (Dolomanov et al., 2009); software used to prepare material for publication: SHELXL97.

We thank Tongling Liang at the Chinese Academy of Sciences for the X-ray crystallographic structure determination.

Supplementary data and figures for this paper are available from the IUCr electronic archives (Reference: FY2092).

\section{References}

Barun, H. O., Ila, H. \& Junjappa, H. (2000). J. Org. Chem. 65, 1583-1587.

Dolomanov, O. V., Bourhis, L. J., Gildea, R. J., Howard, J. A. K. \& Puschmann, H. (2009). J. Appl. Cryst. 42, 339-341.

Huang, Z.-T. \& Liu, Z.-R. (1989). Synth. Commun. 19, 943-958.

Huang, Z.-T. \& Wang, M.-X. (1994). Heterocycles, 37, 1233-1262.

Kuettel, S., Zambon, A., Kaiser, M., Brun, R., Scapozza, L. \& Perozzo, R. (2007). J. Med. Chem. 50, 5833-5839.

Rigaku (2007). CrystalClear. Rigau Corporation, Tokyo, Japan.

Sheldrick, G. M. (2008). Acta Cryst. A64, 112-122. 


\section{supporting information}

Acta Cryst. (2013). E69, o1036 [https://doi.org/10.1107/S1600536813014542]

\section{3,3-Bis(methylsulfanyl)-1-(4-nitrophenyl)prop-2-en-1-one}

\section{Guan-Neng Yu, Jian-Hui Xia, Zhao-Hui Xu, Li-Ben Wang and Chu-Yi Yu}

\section{S1. Comment}

Heterocyclic ketene aminals are important and versatile starting materials for the synthesis of a wide variety of fused heterocycles (Huang \& Wang, 1994). In this paper, we report the crystal structure of the title compound, which is a

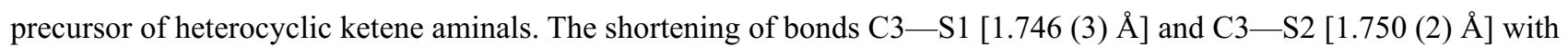
respect to bonds $\mathrm{C} 1-\mathrm{S} 1[1.794(3) \AA]$ and $\mathrm{C} 2-\mathrm{S} 2[1.806(3) \AA]$ is attributed to $d-\pi$ interactions between sulfur and the $\mathrm{C}=\mathrm{C}$ bond. The dihedral angle between 4-nitrophenyl group and $(\mathrm{C} 1, \mathrm{C} 2, \mathrm{~S} 1, \mathrm{~S} 2, \mathrm{C} 3, \mathrm{C} 4, \mathrm{C} 5, \mathrm{O} 1)$ is $35.07(7)^{\circ}$. In the structure of the title compound, molecules are connected through intermolecular $\mathrm{C}-\mathrm{H} \cdots \mathrm{S}$ and $\mathrm{C}-\mathrm{H} \cdots \mathrm{O}$ hydrogen bonding (Table 1) into a three-dimensional network.

\section{S2. Experimental}

The title compound was prepared according to the method of Huang \& Liu (1989). m.p. 435-437 K. MS: m/z=269 $\left(M^{+}\right)$. IR: $1615(\mathrm{C}=\mathrm{O}), 1590(\mathrm{C}=\mathrm{C}), 1512,1345\left(\mathrm{NO}_{2}\right) \mathrm{cm}^{-1 .}{ }^{1} \mathrm{H}-\mathrm{NMR}: \delta=8.33(\mathrm{~d}, 2 \mathrm{H}), 8.07(\mathrm{~d}, 2 \mathrm{H}), 6.73(\mathrm{~s}, 1 \mathrm{H}), 2.60(\mathrm{~s}, 3 \mathrm{H})$, 2.57 (s, 3H) p.p.m.. ${ }^{13} \mathrm{C}-\mathrm{NMR}: \delta=183.5,170.6,149.9,144.9,129.0,124.1,109.0,17.9,15.6$ p.p.m.. Anal. Calc. for $\mathrm{C}_{11} \mathrm{H}_{11} \mathrm{NO}_{3} \mathrm{~S}_{2}$ : C, 49.05; H, 4.12; N, 5.20. Found C, 49.29; H, 4.23; N, 5.36. Single crystals of the title compound suitable for X-ray diffraction analysis were obtained from ethanol solution by slow evaporation after a week.

\section{S3. Refinement}

$\mathrm{H}$ atoms were positioned geometrically and refined using a riding model, with $\mathrm{C}-\mathrm{H}=0.98(\mathrm{C} 1, \mathrm{C} 3)$ or $0.95 \AA$ (C6) and with $U_{\text {iso }}(\mathrm{H})=1.5$ times $U_{\text {eq }}(\mathrm{C})$ (methyl groups) or with $U_{\text {iso }}(\mathrm{H})=1.2$ times $U_{\text {eq }}(\mathrm{C})$ (benzene ring).

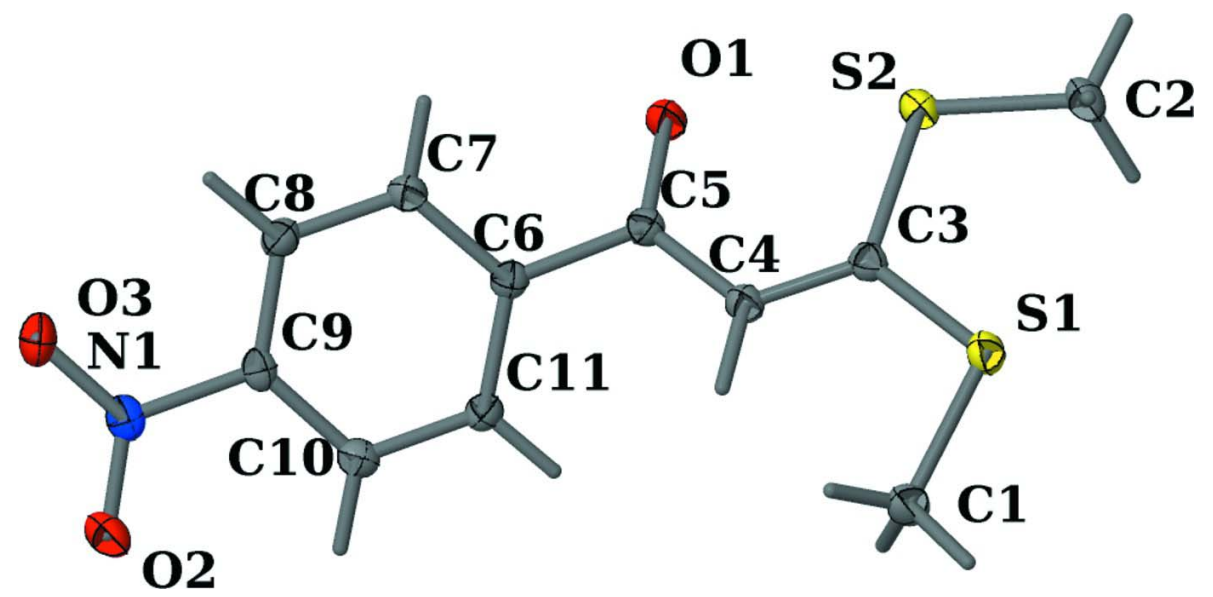

Figure 1

The molecular structure of (I). Displacement ellipsoids are drawn at the $30 \%$ probability level. 


\section{3,3-Bis(methylsulfanyl)-1-(4-nitrophenyl)prop-2-en-1-one}

\section{Crystal data}

$\mathrm{C}_{11} \mathrm{H}_{11} \mathrm{NO}_{3} \mathrm{~S}_{2}$

$M_{r}=269.33$

Triclinic, $P \overline{1}$

$a=7.917(2) \AA$

$b=8.739(2) \AA$

$c=9.574(3) \AA$

$\alpha=70.415(13)^{\circ}$

$\beta=81.985(14)^{\circ}$

$\gamma=73.283(13)^{\circ}$

$V=597.0(3) \AA^{3}$

\section{Data collection}

Saturn724+ CCD

diffractometer

Radiation source: sealed tube

Graphite monochromator

$\omega$ scans at fixed $\chi=45^{\circ}$

Absorption correction: multi-scan

(CrystalClear; Rigaku, 2007)

$T_{\min }=0.581, T_{\max }=1.000$

\section{Refinement}

Refinement on $F^{2}$

Least-squares matrix: full

$R\left[F^{2}>2 \sigma\left(F^{2}\right)\right]=0.055$

$w R\left(F^{2}\right)=0.115$

$S=1.15$

2719 reflections

156 parameters

0 restraints

Primary atom site location: structure-invariant direct methods
$Z=2$

$F(000)=280$

$D_{\mathrm{x}}=1.498 \mathrm{Mg} \mathrm{m}^{-3}$

Mo $K \alpha$ radiation, $\lambda=0.71073 \AA$

Cell parameters from 2392 reflections

$\theta=2.3-32.6^{\circ}$

$\mu=0.44 \mathrm{~mm}^{-1}$

$T=173 \mathrm{~K}$

Plate, yellow

$0.27 \times 0.24 \times 0.05 \mathrm{~mm}$

7843 measured reflections

2719 independent reflections

2394 reflections with $I>2 \sigma(I)$

$R_{\text {int }}=0.053$

$\theta_{\text {max }}=27.5^{\circ}, \theta_{\min }=2.3^{\circ}$

$h=-10 \rightarrow 10$

$k=-11 \rightarrow 11$

$l=-12 \rightarrow 12$

Secondary atom site location: difference Fourier map

Hydrogen site location: inferred from neighbouring sites

$\mathrm{H}$-atom parameters constrained

$w=1 /\left[\sigma^{2}\left(F_{\mathrm{o}}^{2}\right)+(0.0306 P)^{2}+0.3933 P\right]$

where $P=\left(F_{\mathrm{o}}{ }^{2}+2 F_{\mathrm{c}}{ }^{2}\right) / 3$

$(\Delta / \sigma)_{\max }<0.001$

$\Delta \rho_{\max }=0.29 \mathrm{e} \AA^{-3}$

$\Delta \rho_{\min }=-0.23$ e $\AA^{-3}$

\section{Special details}

Geometry. All e.s.d.'s (except the e.s.d. in the dihedral angle between two 1.s. planes) are estimated using the full covariance matrix. The cell e.s.d.'s are taken into account individually in the estimation of e.s.d.'s in distances, angles and torsion angles; correlations between e.s.d.'s in cell parameters are only used when they are defined by crystal symmetry. An approximate (isotropic) treatment of cell e.s.d.'s is used for estimating e.s.d.'s involving 1.s. planes.

Refinement. Refinement of $F^{2}$ against ALL reflections. The weighted $R$-factor $w R$ and goodness of fit $S$ are based on $F^{2}$, conventional $R$-factors $R$ are based on $F$, with $F$ set to zero for negative $F^{2}$. The threshold expression of $F^{2}>\sigma\left(F^{2}\right)$ is used only for calculating $R$-factors(gt) etc. and is not relevant to the choice of reflections for refinement. $R$-factors based on $F^{2}$ are statistically about twice as large as those based on $F$, and $R$ - factors based on ALL data will be even larger.

Fractional atomic coordinates and isotropic or equivalent isotropic displacement parameters $\left(\hat{A}^{2}\right)$

\begin{tabular}{lllll}
\hline & $x$ & $y$ & $z$ & $U_{\text {iso }} * / U_{\text {eq }}$ \\
\hline S1 & $1.24009(8)$ & $0.08728(8)$ & $0.14324(8)$ & $0.03498(19)$ \\
S2 & $1.38394(8)$ & $0.37041(8)$ & $0.12260(7)$ & $0.03303(18)$ \\
O1 & $1.1193(2)$ & $0.6171(2)$ & $0.1933(2)$ & $0.0386(4)$ \\
O2 & $0.2086(2)$ & $0.7642(3)$ & $0.4617(2)$ & $0.0429(5)$
\end{tabular}




$\begin{array}{lllll}\text { O3 } & 0.3377(3) & 0.8672(3) & 0.5789(2) & 0.0518(6) \\ \text { N1 } & 0.3402(3) & 0.7914(3) & 0.4916(2) & 0.0338(5) \\ \text { C1 } & 1.0268(3) & 0.0451(3) & 0.1766(3) & 0.0404(6) \\ \text { H1A } & 0.9467 & 0.1298 & 0.1022 & 0.061^{*} \\ \text { H1B } & 0.9799 & 0.0492 & 0.2759 & 0.061^{*} \\ \text { H1C } & 1.0375 & -0.0668 & 0.1698 & 0.061^{*} \\ \text { C2 } & 1.5600(3) & 0.2069(4) & 0.0782(3) & 0.0398(6) \\ \text { H2A } & 1.5239 & 0.1753 & 0.0001 & 0.060^{*} \\ \text { H2B } & 1.5857 & 0.1086 & 0.1670 & 0.060^{*} \\ \text { H2C } & 1.6660 & 0.2481 & 0.0434 & 0.060^{*} \\ \text { C3 } & 1.2001(3) & 0.2871(3) & 0.1613(3) & 0.0280(5) \\ \text { C4 } & 1.0404(3) & 0.3722(3) & 0.2043(3) & 0.0297(5) \\ \text { H4 } & 0.9447 & 0.3218 & 0.2225 & 0.036^{*} \\ \text { C5 } & 1.0078(3) & 0.5353(3) & 0.2239(3) & 0.0299(5) \\ \text { C6 } & 0.8303(3) & 0.6031(3) & 0.2912(3) & 0.0273(5) \\ \text { C7 } & 0.8213(3) & 0.6942(3) & 0.3879(3) & 0.0309(5) \\ \text { H7 } & 0.9257 & 0.7142 & 0.4083 & 0.037^{*} \\ \text { C8 } & 0.6607(3) & 0.7558(3) & 0.4548(3) & 0.0321(5) \\ \text { H8 } & 0.6540 & 0.8155 & 0.5229 & 0.038^{*} \\ \text { C9 } & 0.5109(3) & 0.7280(3) & 0.4199(3) & 0.0280(5) \\ \text { C10 } & 0.5143(3) & 0.6403(3) & 0.3229(3) & 0.0300(5) \\ \text { H10 } & 0.4088 & 0.6243 & 0.3002 & 0.036^{*} \\ \text { C11 } & 0.6762(3) & 0.5765(3) & 0.2597(3) & 0.0292(5) \\ \text { H11 } & 0.6823 & 0.5139 & 0.1941 & 0.035^{*}\end{array}$

Atomic displacement parameters $\left(\AA^{2}\right)$

\begin{tabular}{lllllll}
\hline & $U^{11}$ & $U^{22}$ & $U^{33}$ & $U^{12}$ & $U^{13}$ & $U^{23}$ \\
\hline S1 & $0.0283(3)$ & $0.0309(3)$ & $0.0462(4)$ & $-0.0060(3)$ & $0.0047(3)$ & $-0.0165(3)$ \\
S2 & $0.0256(3)$ & $0.0382(4)$ & $0.0383(4)$ & $-0.0103(3)$ & $0.0037(3)$ & $-0.0160(3)$ \\
O1 & $0.0320(10)$ & $0.0386(10)$ & $0.0522(12)$ & $-0.0155(8)$ & $0.0104(8)$ & $-0.0227(9)$ \\
O2 & $0.0277(10)$ & $0.0525(12)$ & $0.0472(12)$ & $-0.0118(9)$ & $0.0037(8)$ & $-0.0150(10)$ \\
O3 & $0.0423(12)$ & $0.0650(14)$ & $0.0573(13)$ & $-0.0078(10)$ & $0.0109(10)$ & $-0.0411(12)$ \\
N1 & $0.0305(11)$ & $0.0329(11)$ & $0.0342(12)$ & $-0.0064(9)$ & $0.0031(9)$ & $-0.0090(10)$ \\
C1 & $0.0329(14)$ & $0.0321(13)$ & $0.0599(18)$ & $-0.0101(12)$ & $0.0026(13)$ & $-0.0194(13)$ \\
C2 & $0.0242(13)$ & $0.0458(16)$ & $0.0479(16)$ & $-0.0069(12)$ & $0.0054(11)$ & $-0.0174(13)$ \\
C3 & $0.0272(12)$ & $0.0304(12)$ & $0.0264(12)$ & $-0.0082(10)$ & $-0.0004(9)$ & $-0.0088(10)$ \\
C4 & $0.0274(12)$ & $0.0296(12)$ & $0.0332(13)$ & $-0.0093(10)$ & $0.0022(10)$ & $-0.0108(11)$ \\
C5 & $0.0295(12)$ & $0.0326(13)$ & $0.0303(13)$ & $-0.0114(11)$ & $0.0019(10)$ & $-0.0118(10)$ \\
C6 & $0.0293(12)$ & $0.0252(11)$ & $0.0271(12)$ & $-0.0077(10)$ & $0.0005(9)$ & $-0.0077(10)$ \\
C7 & $0.0270(12)$ & $0.0320(13)$ & $0.0371(14)$ & $-0.0109(10)$ & $0.0023(10)$ & $-0.0139(11)$ \\
C8 & $0.0357(14)$ & $0.0327(13)$ & $0.0323(13)$ & $-0.0111(11)$ & $0.0010(10)$ & $-0.0150(11)$ \\
C9 & $0.0271(12)$ & $0.0268(12)$ & $0.0265(12)$ & $-0.0052(10)$ & $0.0027(9)$ & $-0.0067(10)$ \\
C10 & $0.0275(12)$ & $0.0292(12)$ & $0.0326(13)$ & $-0.0086(10)$ & $-0.0031(10)$ & $-0.0070(10)$ \\
C11 & $0.0304(12)$ & $0.0306(12)$ & $0.0295(12)$ & $-0.0073(10)$ & $-0.0016(10)$ & $-0.0136(10)$ \\
& & & & & & \\
\hline
\end{tabular}


Geometric parameters $\left(\AA,{ }^{\circ}\right)$

\begin{tabular}{|c|c|c|c|}
\hline $\mathrm{S} 1-\mathrm{C} 3$ & $1.746(3)$ & $\mathrm{C} 3-\mathrm{C} 4$ & $1.357(3)$ \\
\hline $\mathrm{S} 1-\mathrm{C} 1$ & $1.794(3)$ & $\mathrm{C} 4-\mathrm{C} 5$ & $1.444(3)$ \\
\hline $\mathrm{S} 2-\mathrm{C} 3$ & $1.750(2)$ & $\mathrm{C} 4-\mathrm{H} 4$ & 0.9500 \\
\hline $\mathrm{S} 2-\mathrm{C} 2$ & $1.806(3)$ & $\mathrm{C} 5-\mathrm{C} 6$ & $1.505(3)$ \\
\hline $\mathrm{O} 1-\mathrm{C} 5$ & $1.236(3)$ & $\mathrm{C} 6-\mathrm{C} 7$ & $1.392(3)$ \\
\hline $\mathrm{O} 2-\mathrm{N} 1$ & $1.223(3)$ & $\mathrm{C} 6-\mathrm{C} 11$ & $1.395(3)$ \\
\hline $\mathrm{O} 3-\mathrm{N} 1$ & $1.223(3)$ & $\mathrm{C} 7-\mathrm{C} 8$ & $1.389(3)$ \\
\hline $\mathrm{N} 1-\mathrm{C} 9$ & $1.473(3)$ & $\mathrm{C} 7-\mathrm{H} 7$ & 0.9500 \\
\hline $\mathrm{C} 1-\mathrm{H} 1 \mathrm{~A}$ & 0.9800 & $\mathrm{C} 8-\mathrm{C} 9$ & $1.381(3)$ \\
\hline $\mathrm{C} 1-\mathrm{H} 1 \mathrm{~B}$ & 0.9800 & $\mathrm{C} 8-\mathrm{H} 8$ & 0.9500 \\
\hline $\mathrm{C} 1-\mathrm{H} 1 \mathrm{C}$ & 0.9800 & $\mathrm{C} 9-\mathrm{C} 10$ & $1.381(3)$ \\
\hline $\mathrm{C} 2-\mathrm{H} 2 \mathrm{~A}$ & 0.9800 & $\mathrm{C} 10-\mathrm{C} 11$ & $1.385(3)$ \\
\hline $\mathrm{C} 2-\mathrm{H} 2 \mathrm{~B}$ & 0.9800 & $\mathrm{C} 10-\mathrm{H} 10$ & 0.9500 \\
\hline $\mathrm{C} 2-\mathrm{H} 2 \mathrm{C}$ & 0.9800 & $\mathrm{C} 11-\mathrm{H} 11$ & 0.9500 \\
\hline $\mathrm{C} 3-\mathrm{S} 1-\mathrm{C} 1$ & $104.01(12)$ & $\mathrm{C} 5-\mathrm{C} 4-\mathrm{H} 4$ & 118.2 \\
\hline $\mathrm{C} 3-\mathrm{S} 2-\mathrm{C} 2$ & $103.83(12)$ & $\mathrm{O} 1-\mathrm{C} 5-\mathrm{C} 4$ & $123.4(2)$ \\
\hline $\mathrm{O} 2-\mathrm{N} 1-\mathrm{O} 3$ & $123.4(2)$ & $\mathrm{O} 1-\mathrm{C} 5-\mathrm{C} 6$ & $119.3(2)$ \\
\hline $\mathrm{O} 2-\mathrm{N} 1-\mathrm{C} 9$ & $118.3(2)$ & $\mathrm{C} 4-\mathrm{C} 5-\mathrm{C} 6$ & $117.2(2)$ \\
\hline $\mathrm{O} 3-\mathrm{N} 1-\mathrm{C} 9$ & $118.3(2)$ & $\mathrm{C} 7-\mathrm{C} 6-\mathrm{C} 11$ & $119.6(2)$ \\
\hline $\mathrm{S} 1-\mathrm{C} 1-\mathrm{H} 1 \mathrm{~A}$ & 109.5 & $\mathrm{C} 7-\mathrm{C} 6-\mathrm{C} 5$ & $118.6(2)$ \\
\hline $\mathrm{S} 1-\mathrm{C} 1-\mathrm{H} 1 \mathrm{~B}$ & 109.5 & $\mathrm{C} 11-\mathrm{C} 6-\mathrm{C} 5$ & $121.8(2)$ \\
\hline $\mathrm{H} 1 \mathrm{~A}-\mathrm{C} 1-\mathrm{H} 1 \mathrm{~B}$ & 109.5 & $\mathrm{C} 8-\mathrm{C} 7-\mathrm{C} 6$ & $120.4(2)$ \\
\hline $\mathrm{S} 1-\mathrm{C} 1-\mathrm{H} 1 \mathrm{C}$ & 109.5 & $\mathrm{C} 8-\mathrm{C} 7-\mathrm{H} 7$ & 119.8 \\
\hline $\mathrm{H} 1 \mathrm{~A}-\mathrm{C} 1-\mathrm{H} 1 \mathrm{C}$ & 109.5 & $\mathrm{C} 6-\mathrm{C} 7-\mathrm{H} 7$ & 119.8 \\
\hline $\mathrm{H} 1 \mathrm{~B}-\mathrm{C} 1-\mathrm{H} 1 \mathrm{C}$ & 109.5 & $\mathrm{C} 9-\mathrm{C} 8-\mathrm{C} 7$ & $118.3(2)$ \\
\hline $\mathrm{S} 2-\mathrm{C} 2-\mathrm{H} 2 \mathrm{~A}$ & 109.5 & $\mathrm{C} 9-\mathrm{C} 8-\mathrm{H} 8$ & 120.9 \\
\hline $\mathrm{S} 2-\mathrm{C} 2-\mathrm{H} 2 \mathrm{~B}$ & 109.5 & $\mathrm{C} 7-\mathrm{C} 8-\mathrm{H} 8$ & 120.9 \\
\hline $\mathrm{H} 2 \mathrm{~A}-\mathrm{C} 2-\mathrm{H} 2 \mathrm{~B}$ & 109.5 & $\mathrm{C} 10-\mathrm{C} 9-\mathrm{C} 8$ & $123.0(2)$ \\
\hline $\mathrm{S} 2-\mathrm{C} 2-\mathrm{H} 2 \mathrm{C}$ & 109.5 & $\mathrm{C} 10-\mathrm{C} 9-\mathrm{N} 1$ & $118.4(2)$ \\
\hline $\mathrm{H} 2 \mathrm{~A}-\mathrm{C} 2-\mathrm{H} 2 \mathrm{C}$ & 109.5 & $\mathrm{C} 8-\mathrm{C} 9-\mathrm{N} 1$ & $118.6(2)$ \\
\hline $\mathrm{H} 2 \mathrm{~B}-\mathrm{C} 2-\mathrm{H} 2 \mathrm{C}$ & 109.5 & $\mathrm{C} 9-\mathrm{C} 10-\mathrm{C} 11$ & $118.0(2)$ \\
\hline $\mathrm{C} 4-\mathrm{C} 3-\mathrm{S} 1$ & $123.37(19)$ & $\mathrm{C} 9-\mathrm{C} 10-\mathrm{H} 10$ & 121.0 \\
\hline $\mathrm{C} 4-\mathrm{C} 3-\mathrm{S} 2$ & $121.71(19)$ & $\mathrm{C} 11-\mathrm{C} 10-\mathrm{H} 10$ & 121.0 \\
\hline $\mathrm{S} 1-\mathrm{C} 3-\mathrm{S} 2$ & $114.92(14)$ & $\mathrm{C} 10-\mathrm{C} 11-\mathrm{C} 6$ & $120.8(2)$ \\
\hline $\mathrm{C} 3-\mathrm{C} 4-\mathrm{C} 5$ & $123.6(2)$ & $\mathrm{C} 10-\mathrm{C} 11-\mathrm{H} 11$ & 119.6 \\
\hline $\mathrm{C} 3-\mathrm{C} 4-\mathrm{H} 4$ & 118.2 & $\mathrm{C} 6-\mathrm{C} 11-\mathrm{H} 11$ & 119.6 \\
\hline $\mathrm{C} 1-\mathrm{S} 1-\mathrm{C} 3-\mathrm{C} 4$ & $5.0(3)$ & $\mathrm{C} 5-\mathrm{C} 6-\mathrm{C} 7-\mathrm{C} 8$ & $-178.6(2)$ \\
\hline $\mathrm{C} 1-\mathrm{S} 1-\mathrm{C} 3-\mathrm{S} 2$ & $-176.01(14)$ & $\mathrm{C} 6-\mathrm{C} 7-\mathrm{C} 8-\mathrm{C} 9$ & $-1.6(4)$ \\
\hline $\mathrm{C} 2-\mathrm{S} 2-\mathrm{C} 3-\mathrm{C} 4$ & $178.0(2)$ & $\mathrm{C} 7-\mathrm{C} 8-\mathrm{C} 9-\mathrm{C} 10$ & $0.7(4)$ \\
\hline $\mathrm{C} 2-\mathrm{S} 2-\mathrm{C} 3-\mathrm{S} 1$ & $-0.99(17)$ & $\mathrm{C} 7-\mathrm{C} 8-\mathrm{C} 9-\mathrm{N} 1$ & $179.4(2)$ \\
\hline $\mathrm{S} 1-\mathrm{C} 3-\mathrm{C} 4-\mathrm{C} 5$ & $178.02(19)$ & $\mathrm{O} 2-\mathrm{N} 1-\mathrm{C} 9-\mathrm{C} 10$ & $-0.3(3)$ \\
\hline $\mathrm{S} 2-\mathrm{C} 3-\mathrm{C} 4-\mathrm{C} 5$ & $-0.9(4)$ & $\mathrm{O} 3-\mathrm{N} 1-\mathrm{C} 9-\mathrm{C} 10$ & $179.1(2)$ \\
\hline $\mathrm{C} 3-\mathrm{C} 4-\mathrm{C} 5-\mathrm{O} 1$ & $5.3(4)$ & $\mathrm{O} 2-\mathrm{N} 1-\mathrm{C} 9-\mathrm{C} 8$ & $-179.0(2)$ \\
\hline $\mathrm{C} 3-\mathrm{C} 4-\mathrm{C} 5-\mathrm{C} 6$ & $-172.2(2)$ & $\mathrm{O} 3-\mathrm{N} 1-\mathrm{C} 9-\mathrm{C} 8$ & $0.4(3)$ \\
\hline
\end{tabular}




$\begin{array}{llll}\mathrm{O} 1-\mathrm{C} 5-\mathrm{C} 6-\mathrm{C} 7 & -34.5(3) & \mathrm{C} 8-\mathrm{C} 9-\mathrm{C} 10-\mathrm{C} 11 & 0.7(4) \\ \mathrm{C} 4-\mathrm{C} 5-\mathrm{C} 6-\mathrm{C} 7 & 143.0(2) & \mathrm{N} 1-\mathrm{C} 9-\mathrm{C} 10-\mathrm{C} 11 & -178.0(2) \\ \mathrm{O} 1-\mathrm{C} 5-\mathrm{C} 6-\mathrm{C} 11 & 145.8(2) & \mathrm{C} 9-\mathrm{C} 10-\mathrm{C} 11-\mathrm{C} 6 & -1.2(4) \\ \mathrm{C} 4-\mathrm{C} 5-\mathrm{C} 6-\mathrm{C} 11 & -36.7(3) & \mathrm{C} 7-\mathrm{C} 6-\mathrm{C} 11-\mathrm{C} 10 & 0.4(4) \\ \mathrm{C} 11-\mathrm{C} 6-\mathrm{C} 7-\mathrm{C} 8 & 1.1(4) & \mathrm{C} 5-\mathrm{C} 6-\mathrm{C} 11-\mathrm{C} 10 & -180.0(2)\end{array}$

Hydrogen-bond geometry $\left(\AA,{ }^{\circ}\right)$

\begin{tabular}{lllll}
\hline$D-\mathrm{H} \cdots A$ & $D-\mathrm{H}$ & $\mathrm{H} \cdots A$ & $D \cdots A$ & $D-\mathrm{H}^{\cdots} A$ \\
\hline $\mathrm{C} 11-\mathrm{H} 11 \cdots \mathrm{S} 2^{\mathrm{i}}$ & 0.95 & 2.93 & $3.614(3)$ & 130 \\
$\mathrm{C} 8-\mathrm{H} 8 \cdots 3^{\mathrm{ii}}$ & 0.95 & 2.63 & $3.204(3)$ & 119 \\
$\mathrm{C} 2-\mathrm{H} 2 B \cdots \mathrm{O} 3^{\mathrm{iii}}$ & 0.98 & 2.68 & $3.297(4)$ & 122 \\
$\mathrm{C} 10-\mathrm{H} 10 \cdots{ }^{\mathrm{ii}}$ & 0.95 & 2.66 & $3.602(3)$ & 171 \\
$\mathrm{C} 1-\mathrm{H} 1 C \cdots{ }^{\mathrm{v}}$ & 0.98 & 2.59 & $3.551(3)$ & 167 \\
$\mathrm{C} 7-\mathrm{H} 7 \cdots \mathrm{O} 2^{\mathrm{vi}}$ & 0.95 & 2.55 & $3.499(3)$ & 179 \\
\hline
\end{tabular}

Symmetry codes: (i) $-x+2,-y+1,-z$; (ii) $-x+1,-y+2,-z+1$; (iii) $-x+2,-y+1,-z+1$; (iv) $x-1, y, z$; (v) $x, y-1, z$; (vi) $x+1, y, z$. 